\title{
Étude du phénomène d'écrasement des grains de schiste au compactage
}

\section{B. MELBOUCI}

Université

Mouloud Mammeri

Faculté du Génie

de la Construction

Laboratoire Géomatériaux

Environnement

et Aménagement BP 17 RP

15000 Tizi Ouzou (Algérie) bmelbouci@yahoo.fr

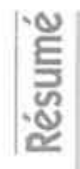

L'amélioration des matériaux par compactage est directement liée non seulement à la granulométrie des matériaux à traiter mais aussi à la forme de leurs grains, leur angularité et à la teneur en eau contenue dans ces derniers. Cet article présente les résultats d'une étude expérimentale de deux essais: Proctor et CBR et de leur influence sur le phénomène d'écrasement des graíns de trois matériaux locaux schisteux (schiste argileux, schiste satiné et schiste tacheté). Cet écrasement est quantifié par deux paramètres: le coefficient d'uniformité $C_{n}$ et le taux d'écrasement $\mathrm{B}_{10}$.

Ces résultats montrent que le taux de rupture des grains est mis en évidence par l'étalement des courbes granulométriques qui évoluent en fonction de l'énergie de compactage. Le coefficient d'uniformité $C_{4}$ et le facteur $B_{10}$ confirment que cette évolution est fonction des classes granulaires, de la présence d'eau et de la dureté des grains.

Mots-clés : grain de schiste, compactage, essai Proctor, essai CBR, écrasement.

\section{Crushing phenomenon of the grains schist during compaction}

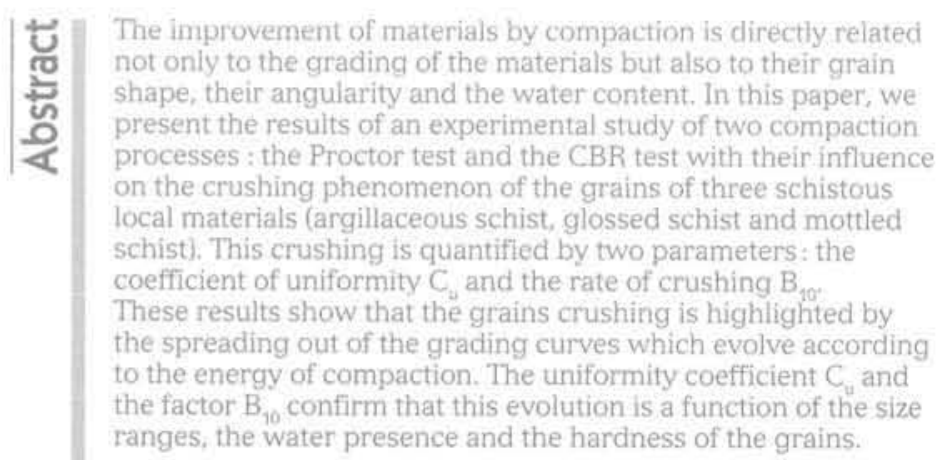

Key words: schist grain, compaction. Proctor test, CBR test, crushing: 


\section{NOTATIONS}

$B_{10}$ : facteur mesurant le taux d'écrasement

$\mathrm{C} \quad$ : compacité relative

CBR : capacity bearing ratio

$\mathrm{C}_{u} \quad$ : coefficient d'uniformité

e : indice des vides

G : gonflement

LA : essai Los Angeles

MDE : essai micro-Deval

n : porosité

$\mathrm{Sr}$ : degré de saturation

W : teneur en eau

$W_{\text {pip }}$ : teneur en eau optimum

$\gamma_{d}$ : poids spécifique sec

$\gamma_{\mathrm{s}} \quad$ : poids spécifique des grains solides

\section{1}

\section{Introduction}

Dans la présente étude, nous nous intéressons au matériau schiste qui se trouve en abondance dans la région de la Grande Kabylie dans l'optique d'une éventuelle utilisation dans le domaine du génie civil. Afin d'avoir une connaissance étendue de ce matériau, notre choix s'est porté sur trois types différents de schiste parmi les plus répandus dans la région. Les échantillons ainsi utilisés intègrent diverses qualités de schiste s'étendant des matériaux durs (schiste tacheté) aux materiaux moins durs (schiste argileux).

Les grains formant les trois types de schiste ont une composition minéralogique, une forme et des résistances individuelles différentes. En outre, l'énergie nécessaire pour obtenir à partir d'un grain d'autres grains de taille inférieure est plus ou moins importante. La rupture des grains est donc liée non seulement aux propriétés physiques de ces derniers mais aussi aux chemins de contraintes appliqués. Ainsi diverses questions ont été soulevées pour connaître si réellement il existait une régularité dans le processus de rupture pour divers chemins de contraintes. C'est pourquoi, notre campagne d'essais porte sur l'essai Proctor et l'essai CBR de ces matériaux.

Le compactage aura pour effet de modifier l'arrangement des grains entre eux de façon à obtenir une meilleure compacité laquelle entraîne une diminution plus ou moins rapide du volume des vides. Le compactage suppose l'emploi d'engins lourds suffisamment puissants pour vaincre les forces de frottement entre les grains. Ces engins développent une énergie dite « énergie de compactage». Les grains se cassent au contact des uns et des autres par frottement ou par chocs et ils se transforment en grains de petites dimensions, Cette modification de la granularité est augmentée par la présence d'eau. Lors du compactage, les grains sont serrés, leurs mouvements sont réduits, donc l'écrasement diminue.

Pour mieux caractériser le comportement de ces matériaux, ils sont soumis à deux chemins de contrainte différents: l'essai Proctor sous des énergies élevées allant jusqu'à 150 coups et l'essai CBR. Les grains ont été sélectionnés pour constituer trois classes granulométriques $0 / 6-6 / 10$ et $10 / 14$.
Dans cette étude, nous mettrons l'accent essentiellement sur l'influence des trois paramètres suivants : - la classe granulométrique $(0 / 6 ; 6 / 10$ et $10 / 14)$;

- la présence ou non d'eau:

- la compacité du matériau.

\section{2}

\section{Identification des trois types de schiste}

Pour mener cette étude, des échantillons relatifs à trois types de schistes ont été prélevés, à savoir :

- des roches de schistes argileux qui sont à la limite du domaine du métamorphisme; elles sont encore considérées comme des roches sédimentaires;

- des roches de schistes satinés à deux micas qui montrent des recristallisations minérales plus nettes. Ce sont des roches ayant acquis une schistosité sous l'influence de contraintes tectoniques, de teinte généralement grise présentant des surfaces blanchâtres nacrées ou satinées dues à la présence de feuillets de séricite, Ces roches sont aussi appelées séricitoschistes

- des roches de schistes tachetés qui sont des schistes dans lesquels le métamorphisme de contact (haute température et basse pression) a développé des minéraux néoformes qui se concentrent en agrégats ayant l'aspect de taches (porphyroblastes). La schistosité est en général acquise antérieurement à l'intrusion des roches magmatiques, mais elle peut aussi être liée à la mise en place de l'intrusion. Ces roches sont, en géné$\mathrm{ral}$, de couleur grise à noire.

Ces schistes sont extraits sur des sites situés à moins de 10 kilomètres au nort-est de la ville de Tizi Ouzou sur les flancs longeant l'oued Sebaou. Le relief des sites est accidenté avec des côtes aux altitudes de $100 \mathrm{~m}$ et $150 \mathrm{~m}$. La colonne synthétique des différents ensembles tectonométamorphiques est représentée en figure 1. Les sites ont été choisis pour poursuivre l'étude effectuée par l'Office national de la recherche géologique et minière (ORGM) de Tizi Ouzou. Les trois types de schistes étudiés sont de nature minéralogique différente.
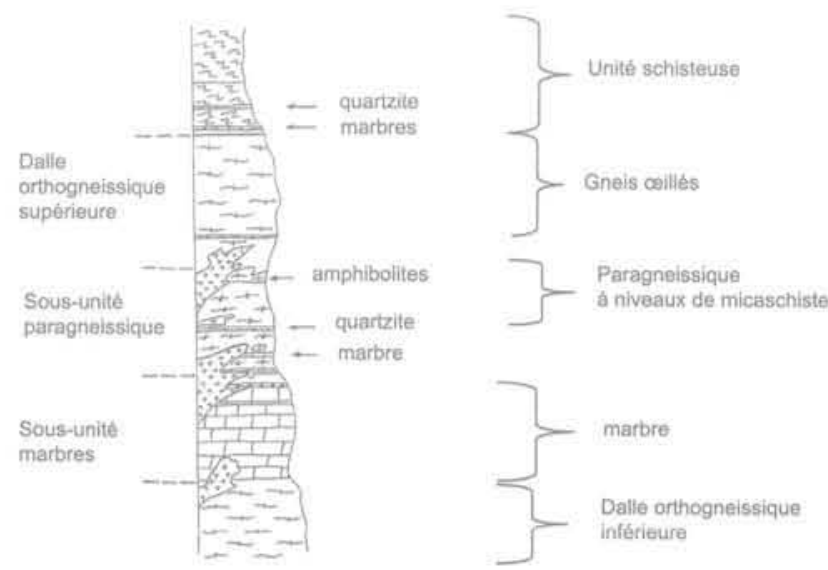

FIG.1 Colonne synthétique des différents ensembles tectonométamorphiques du site de l'extraction des échantillons. 


\section{Étude pétrographique}

L'observation macroscopique des blocs extraits révèle que l'aspect des échantillons est homogène. Ce sont des blocs de roches qui se caractérisent par une couleur : - marron clair pour l'échantillon de schiste argileux; - noire mouchetée de mica pour l'échantillon de schiste satiné à deux micas :

- noire à nuances verdâtres pour l'échantillon de schiste tacheté.

Les blocs utilisés ont été soumis au concassage manuel, la granulométrie obtenue est de 0/20 mm. Ainsi les grains obtenus présentent des angularités (forme angulaire). La densité des grains dépend de leur composition minéralogique et de leur résistance à l'altération.

L'étude pétrographique a été réalisée dans un laboratoire spécialisé de l'Office national de la recherche géologique et minière dit Centre de recherche et du développement (CRD) de Boumerdès. Il s'agit d'études de lames minces dont le but est d'identifier les caractéristiques pétrographiques et minéralogiques des schistes et d'évaluer leur importance relative permettant ainsi de mieux comprendre leur comportement. Les différents minéraux obtenus sont décrits pour les trois schistes. Ainsi, le schiste argileux, de texture schisteuse, est composé des minéraux suivants : 30 à $33 \%$ de quartz, 40 à $45 \%$ de mica, 10 à $15 \%$ de plagioclase et de 5 à $6 \%$ d'oxyde de fer. Le schiste satiné, de texture schisteuse et de structure granole pyroclastique, est composé de 45 à $50 \%$ de quartz, 20 à $25 \%$ de biotite, 20 à $25 \%$ de muscovite et 20 à $25 \%$ de tourmaline. Enfin, le schiste tacheté, de texture massive et compacte et de structure hématoblastique, est composé de 50 à $55 \%$ d'amphiboles, 20 à $25 \%$ de ferro-actinote, 4 à $5 \%$ de calcite et 8 à $10 \%$ de chlorite.

L'étude pétrographique réalisée révèle les points suivants. Il n'existe pas de traces d'argile dans les trois types de schistes analysés. Ceci s'explique par le fait que les schistes sont des roches métamorphiques (non sédimentaires). Elles contiennent plutôt quelques minéraux issus de la transformation directe de l'argile telle que la chlorite (matériaux très instables) ou indirecte tels que les micas (biotite ou muscovite) ou le plagioclase (feldspath, en général) puisque l'argile se transforme avant en chlorite ou en séricite. L'échantillon de schiste argileux contient entre 30 et $33 \%$ de minéraux stables (quartz) et 50 à $60 \%$ de minéraux instables (plagioclase et mica). L'échantillon de schiste satiné contient 65 à $75 \%$ de minéraux stables (quartz et tourmaline) et 40 à $50 \%$ de minéraux instables (micas : biotite et muscovite). L'échantillon de schiste tacheté contient 70 à $80 \%$ de minéraux stables (amphibole et ferro-actinote) et 12 à $15 \%$ de minéraux très instables (calcite et chlorite).

\section{Caractéristiques d'identification}

Après avoir concassé manuellement des blocs de schistes, des échantillons ont été soumis au séchage dans une étuve à la température de $105^{\circ} \mathrm{C}$ pendant 24 heures. Par la suite, une étude d'identification a été réalisée au laboratoire. Les grains ainsi obtenus sont de type très anguleux (Fig. 2).
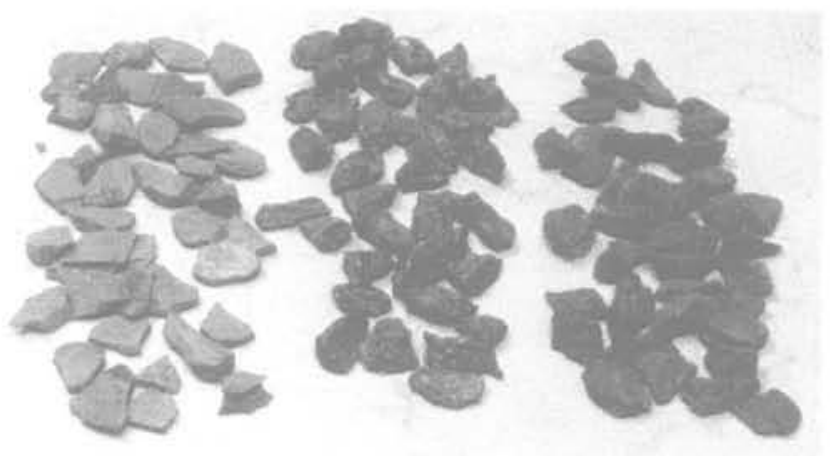

FG. 2 Grains de schistes étudiés. De gauche à droite: schiste argileux, schiste satiné et schiste tacheté.

Les principales caractéristiques physiques des échantillons étudiés sont récapitulées dans les tableaux I et II. Notons que les valeurs présentées dans le tableau I sont des valeur's moyennes obtenues d'une série de trois échantillons.

TABLEAUI Caractéristiques physiques des schistes étudiés.

\begin{tabular}{l|c|c|c|c|c|c} 
Echantillon & $W(\%)$ & $\left.\gamma / \mathrm{m}^{3}\right)$ & $\begin{array}{c}\gamma \\
\left(\mathrm{KN} / \mathrm{m}^{3}\right)\end{array}$ & e & $\operatorname{Sr}(\%)$ & $\mathrm{n}$ \\
\hline Schiste argileux & 6,18 & 22,7 & 26,9 & 0,18 & 89,86 & 0,15 \\
\hline Schiste satiné & 4,96 & 23,4 & 27,9 & 0,19 & 72,07 & 0,16 \\
\hline Schiste tacheté & 5,15 & 24,3 & 28,7 & 0,18 & 81,66 & 0,15 \\
\hline
\end{tabular}

Les courbes Proctor modifié de la figure 3 ont une allure légèrement aplatie, ce qui nous permet de dire que les matériaux sont peu sensibles à l'eau. L'échantillon de schiste tacheté a présenté des valeurs de poids spécifiques les plus élevées contrairement à l'échantillon de schiste argileux. Ceci est lié à sa texture massive et compacte en plus de la présence d'un pourcentage important d'amphibole (50 à $55 \%$ ) connue par sa masse volumique absolue très élevée $\left(32 \mathrm{kN} / \mathrm{m}^{3}\right)$. Cette texture fournit un indice des vides (e) très faible.

TABLEAUII Caractéristiques Proctor, CBR, Los Angeles et micro-Deval en présence d'eau pour les schistes étudiés.

\begin{tabular}{|c|c|c|c|c|c|c|}
\hline & \multicolumn{2}{|c|}{ Proctor madifiê } & \multicolumn{2}{|c|}{ Indice (BR $(\%)$ à } & \multicolumn{2}{|c|}{ Résistance aux chocs et à l'usure } \\
\hline & $\begin{array}{c}\left(\gamma_{\mathrm{j}}\right)_{\mathrm{opt}} \\
\left(\mathrm{kN} / \mathrm{m}^{3}\right)\end{array}$ & $\begin{array}{l}W_{(\%)} \\
(\%)^{\prime}\end{array}$ & $\frac{92 \%}{\text { de compacité }}$ & $\begin{array}{c}98 \% \\
\text { de compacitê }\end{array}$ & $\begin{array}{l}\text { Coefficient } \\
\text { Los Angeles }\end{array}$ & $\begin{array}{l}\text { Coefficient } \\
\text { micro-Deval }\end{array}$ \\
\hline Schiste argileux & 21,0 & 7,80 & 5 & 13 & 75,8 & 38 \\
\hline Schiste satiné & 22,1 & 6,50 & 6 & 25 & 29,2 & 25 \\
\hline Schiste tacheté & 22,6 & 7,10 & 10 & 38 & 35,2 & 24 \\
\hline
\end{tabular}




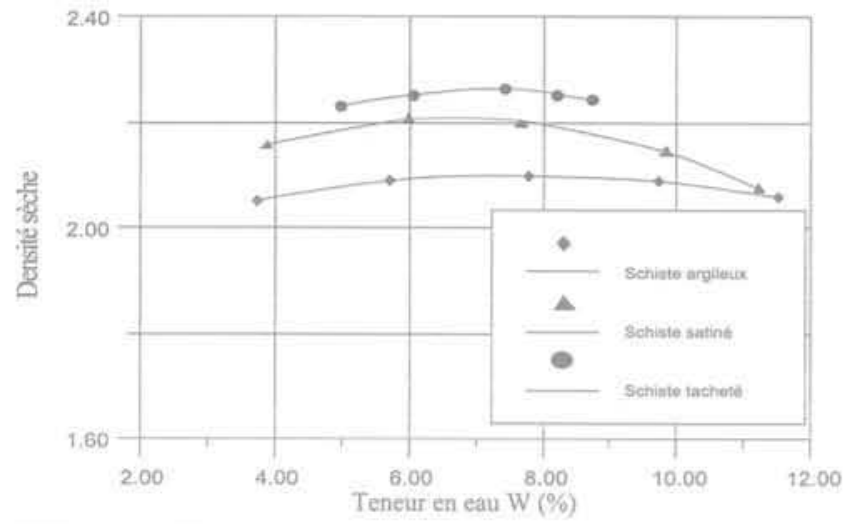

Fig. 3 Courbes de l'essai Proctor modifié des trois schistes.

Le poids volumique $\left(\gamma_{\mathrm{d}}\right)_{\text {o }}$ augmente avec le degré de compacité de la structure interne des matériaux, de la densité des minéraux majeurs les constituant et des pourcentages relatifs de ceux-ci. Tandis que la teneur en eau optimale $\left(w_{\text {opt }}(\%)\right)$ dépend du pourcentage des minéraux instables présents et de leur degré d'instabilité (les minéraux instables sont les plus altérables). En effet, sous des conditions climatiques identiques et pendant une durée fixée, les minéraux n'ont pas tous le même degré d'altérabilité. En respectant les mêmes conditions d'altérabilité, Zumberg, cité dans Lade et Yamamuro et al, (1996), classe les minéraux; ainsi les quartz sont des minéraux très stables. La muscovite, l'orthoclase, la biotite, l'amphibole, le pyroxène, le plagioclase et l'olivine sont des minéraux peu stables.

L'échantillon de schiste argileux a donné les plus grandes valeurs de $W_{\text {nor }}$ le schiste satiné les plus faibles. Le gonflement (Tableau III) et la teneur en eau, augmentent avec le pourcentage des minéraux instables présents dans les échantillons:

\section{3}

\section{Essai CBR sur les schistes étudiés}

Les essais CBR sont effectués sur les échantillons des trois matériaux schisteux compactés à la teneur en eau optimum obtenue par l'essai Proctor modifié, Ils sont ensuite poinçonnés par un piston. Au cours de ces essais, on a constaté que c'est essentiellement le compactage qui permet d'améliorer la portance de ces matériaux et le pouvoir portant de ces derniers est d'autant meilleur que le CBR est plus grand.
Les principaux résultats obtenus à partir des essais CBR sont regroupés dans le tableau III.

L'échantillon tacheté présente un indice CBR, à $98 \%$ de compacité par rapport à la compacité optimale, supérieur à 35, un Los Angeles (L.A) et un micro-Deval en présence d'eau (MDE) inférieur à 40 (Tableau II) et un gonflement nettement inférieur à 1\% (Tableau III). Ce type de granulats peut donc être incorporé dans les corps de chaussée conformément au cahier de prescriptions spéciales (1995). L'échantillon de schiste argileux a enregistré les valeurs les plus élevées du gonflement par rapport aux autres échantillons (Tableau III). En effet, le gonflement et la teneur en eau augmentent avec le pourcentage des minéraux instables présents dans les échantillons. Pour une compacité de l'ordre de $98 \%$, l'indice CBR du schiste argileux est de l'ordre de 13 (Tableau II), indice relativement faible pour une éventuelle utilisation dans les corps de chaussée. Quant au schiste satiné, il présente une valeur intermédiaire parmi les échantillons testés qui est de l'ordre de 25 (Tableau II). Ce schiste peut répondre à certaines utilisations particulièrement dans le domaine routier.

\section{Écrasement des grains}

Il existe pour un granulat, deux types d'évolution possible sous l'action de sollicitations mécaniques : il se fragmente en donnant des éléments de toutes dimensions ou bien il s'use par frottement en donnant essentiellement des éléments fins. Ramamurthy (1969) est le premier auteur à présenter une classification des ruptures des grains qui a été synthétisée par Guyon et Troadec (1994) qui en distinguent trois modes de rupture: la fracture, l'écaillage et l'abrasion. Ils en donnent la définition. La fracture concerne un grain qui se casse pour donner de nouveaux grains de taille sensiblement égale ou inférieure à la taille du grain d'origine. L'écaillage ou l'attrition se produit ainsí: un grain se casse pour donner un grain de taille légèrement inférieure et voire plusieurs grains de plus petite taille. L'abrasion génère un grain ayant sensiblement la même taille que le grain d'origine mais avec une production de particules très fines.

De nombreux chercheurs (Biarez et Hicher, 1997; Marsal, 1977; Cambou, 1972; Lade et Yamamuro et al., 1996; McDowell et Bolton, 1998) se sont penchés sur le phénomène de rupture des grains et ont permis de

TABLEAU iil Influence du gonflement en fonction de la compacité.

\begin{tabular}{|c|c|c|c|c|c|c|}
\hline \multirow[t]{2}{*}{ Intensité de compactage } & \multicolumn{2}{|c|}{ Schiste argileux } & \multicolumn{2}{|c|}{ Schiste satiné } & \multicolumn{2}{|c|}{ Schiste tacheté } \\
\hline & $G(\%)$ & $\mathrm{C}(\%)$ & $G(\%)$ & $\mathrm{C}(\%)$ & $G(\%)$ & $\mathrm{C}(\%)$ \\
\hline $\begin{array}{c}55 \text { coups/couche } \\
\left(w_{\text {ogt }}+2 \%\right)\end{array}$ & 0,70 & 98.8 & 0,29 & 99,3 & 0,19 & 96,6 \\
\hline $\begin{array}{c}55 \text { coups/couche } \\
\left(w_{\text {opp }}\right)\end{array}$ & 0,49 & 10,0 & 0,08 & 97,9 & 0,28 & 99,6 \\
\hline $\begin{array}{c}55 \text { coups/couche } \\
\left(w_{\text {ogt }}-2 \%\right)\end{array}$ & 0,32 & 100,0 & 0,06 & 98,1 & 0,22 & 99,7 \\
\hline $\begin{array}{c}25 \text { coups/couche } \\
\left.\text { ( } \mathrm{w}_{\mathrm{opk}}\right)\end{array}$ & 0,71 & 98,0 & 0,08 & 94,4 & 0,24 & 96,2 \\
\hline $\begin{array}{c}10 \text { coups/couche } \\
\text { ( } \mathrm{w}_{\text {opt }} \text { ) }\end{array}$ & 0.39 & 93,8 & 0,03 & 89,6 & 0,12 & 91,2 \\
\hline
\end{tabular}


comprendre comment ce phénomène se manifeste et quels sont les facteurs qui permettent de quantifier la rupture des grains.

En pratique, la rupture des grains se produit à grande échelle durant le compactage des terrains, particulièrement au niveau des barrages, au-dessous des pointes des pieux, etc., ou durant les essais menés en laboratoire, par exemple lorsque les granulats sont soumis à des contraintes de compression ou de cisaillement. L'importance de cette rupture dépend de la forme, de la résistance propre de chaque grain (composition minéralogique et structure cristalline), de la compacité des matériaux, de l'effet de l'eau, de l'intensité des contraintes appliquées aux points de contact et du chemin des contraintes.

Quand les grains sont solides, durs et assez arrondis, ils peuvent supporter des contraintes élevées sans s'écraser. Lorsqu'ils sont fragiles, ils subissent une fragmentation sous l'effet des contraintes appliquées, résultant du cisaillement des aspérités et de la fissuration des grains (Yamamuro et al., 1996)

L'objectif de cette étude est de quantifier le degré d'écrasement des grains de trois types de schiste: tacheté, argileux et satiné, en comparant les courbes granulométriques obtenues avant et après différents essais Proctor pour les classes granulaires $0 / 6,6 / 10$ et 10/14; et ce pour des états sec lâche et humide et en fonction de l'énergie de compactage.

Hagerty et al. (1993) ont observé trois phases de comportement résultant d'une compression: diminution de volume de l'échantillon due au réarrangement des grains sous faibles contraintes, écrasement des grains et réarrangement sous fortes contraintes.

L'écrasement des grains apparait quand les contraintes imposées aux grains qui composent le sol dépassent leur résistance propre. La compression s'effectue tout d'abord avec un réarrangement, ensuite l'écrasement des grains se produit en commençant par les plus gros et les plus fragiles. La rupture des grains génère une évolution de la granulométrie du matériau, évolution qui permet d'obtenir une compacité plus grande et un indice des vides plus faible. Graduellement, ces fragmentations mènent à une réduction de l'effort moyen appliqué entre les grains. Cette réduction de l'effort diminue le taux d'écrasement méme si l'effort vertical augmente. Ce changement de comportement mène à une augmentation de la rigidité de l'échantillon. L'écrasement non seulement brise les grains faibles mais améliore les performances d'un matériau èn diminuant sa compressibilité et sa perméabilité.

\section{4}

\section{Résultats et interprétation}

Une des méthodes la plus simple qui permet d'étudier l'écrasement des grains est la comparaison des courbes granulométriques obtenues avant et après les différents essais Proctor modifié réalisés sur les trois classes granulaires retenues pour l'étude. Les essais Proctor montrent qu'il y a un grand changement de la structure granulaire en fonction du nombre de coups, de la présence ou non d'eau et de la classe granulaire d'origine. Il est important de noter que toutes les tailles des grains sont concernées par le phénomène de rupture.
L'énergie de compactage ou le nombre de coups influent peu sur la classe granulaire $0 / 6$. On constate même une légère production de fines qui reflète une bonne résistance à l'écrasement de cette granulométrie. En effet, les grains pour cette fraction sont petits et donc plus sains et plus résistants. Pour les classes $6 / 10$ et 10/14, l'écrasement devient plus important avec l'augmentation de l'énergie de compactage.

En présence d'eau, l'écrasement est légèrement plus important pour les grains de taille élevée. En effet, c'est à partir d'une taille supérieure à $6 \mathrm{~mm}$ (cas de la classe 6/10) que l'eau commence à avoir une influence importante sur la rupture des grains. Ainsi on a obtenu des étalements plus marqués des courbes granulométriques et le coefficient d'uniformité a évolué par des valeurs parfois importantes de l'état sec lâche à l'état humide en fonction de la nature des matériaux. L'eau en entourant les grains de cette classe pénètré dans les microfissures et rend les zones les plus fragiles moins consistantes, ceci constitue donc des croûtes facilement dégradables. Ceci confirme les résultats de Miura et O'Hara (1979) qui ont montré que la résistance individuelle en présence d'eau des grains devient plus faible, ce qui conduit à un étalement granulométrique important.

Pour une énergie de compactage fixée à 100 coups (Fig. 4), les matériaux présentent un degré d'écrasement différent. Les courbes granulométriques sont plus étalées pour le schiste argileux, donc la rupture des grains est plus importante pour ces derniers. A l'état humide, l'étalement de ces courbes est plus prononcé (Fig. 5). En fait, l'influence de l'eau sur la dégradation diffère d'un matériau à un autre en fonction de la résistance individuelle de ces derniers (cas du schiste satiné, Fig. 6). Plus les grains sont résistants, moindre est l'effet de l'eau.

Les essais Proctor ont prouvé que l'écrasement des grains augmente considérablement avec l'augmentation du nombre de coups et de manière significative avec la taille des grains de schiste argileux (Fig. 7). Cet écrasement, particulièrement pour la classe 10/14, est d'autant plus important que le matériau est humide pour des énergies de compactage élevées, supérieures à 50 coups (Fig. 5). Au fur et à mesure que la densité relative augmente, la rupture des grains diminue ceci peut s'expliquer par le fait qu'avec plus de particules entourant chaque grain, l'effort moyen de contact tend à diminuer.

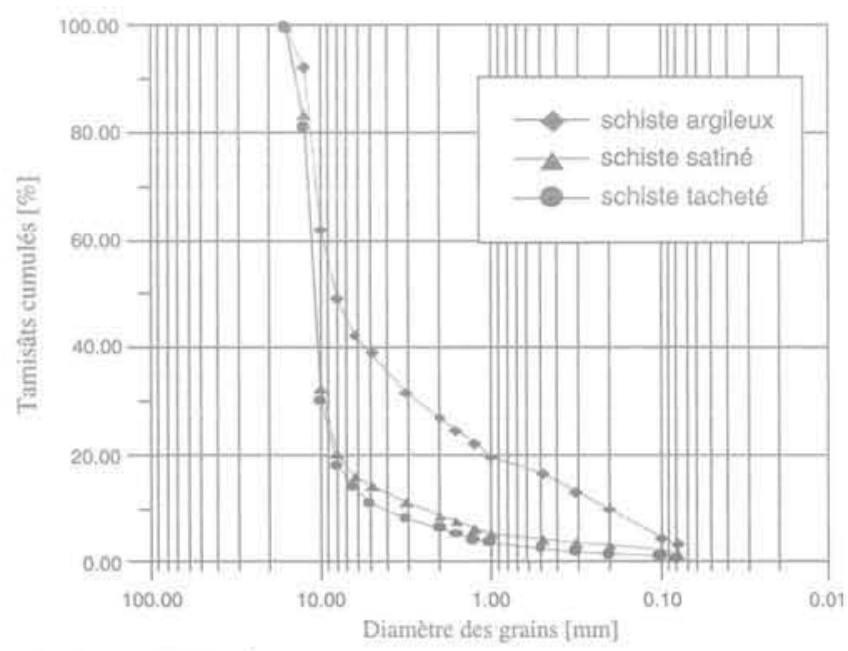

FIG.4 Étalement granulométrique de la classe granulaire 10/14, à l'essai Proctor à l'état sec pour une énergie de compactage équivalente à 100 coups. 


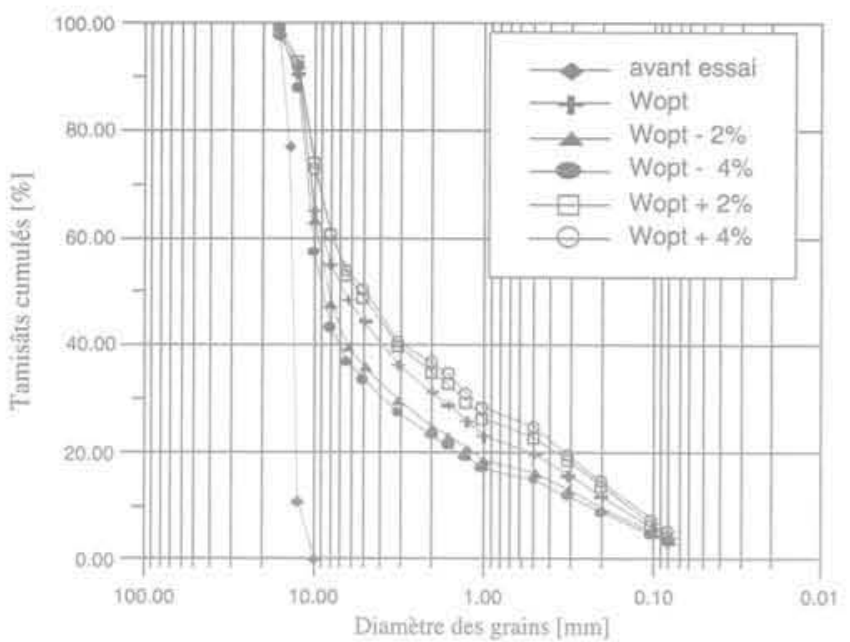

FIG. 5 Influence de la teneur en eau sur le schiste argileux de classe granulaire $10 / 14$, pour une énergie de compactage de 75 coups.

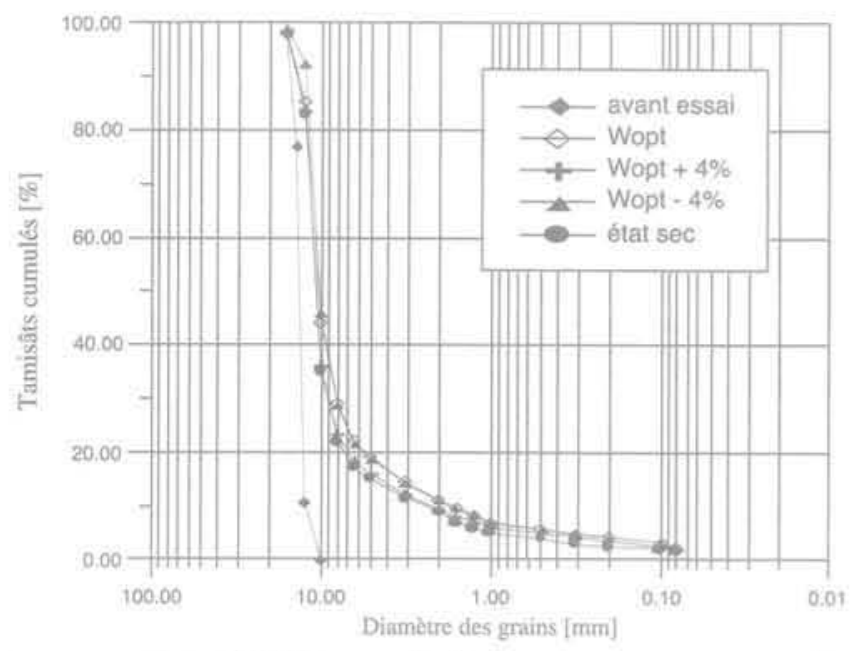

FG. 6 Influence de la teneur en eau sur la dégradation des grains du schiste satiné de classe granulaire $10 / 14$, pour une énergie de compactage de 75 coups.

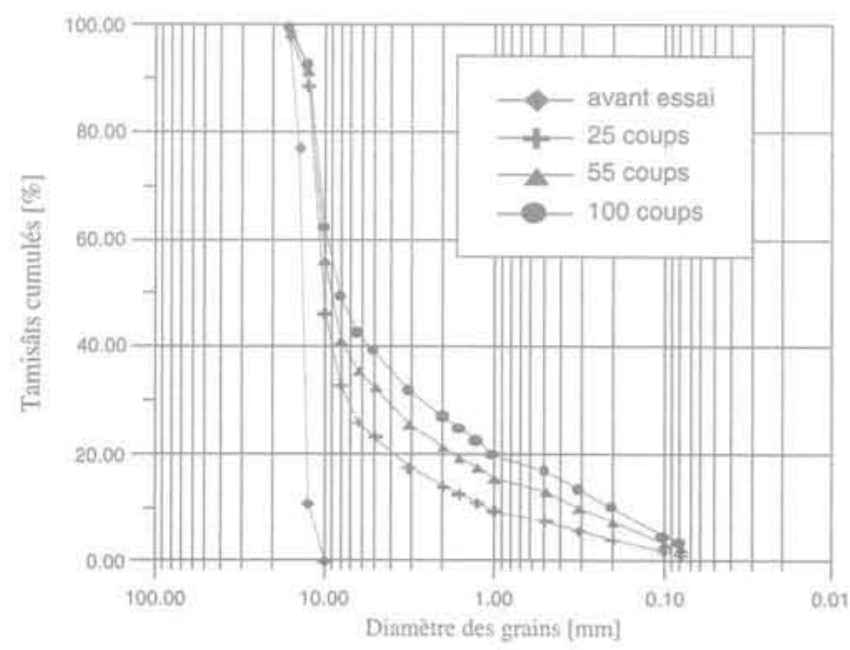

FIG.7 Influence de l'énergie de compactage sur la dégradation des grains du schiste argileux de classe granulaire $10 / 14$ à l'état sec lâche.
Les principaux résultats obtenus à l'essai Proctor ont montré que le schiste satiné et le schiste tacheté sont les matériaux les plus résistants au compactage et celui qui se dégrade le plus est le schiste argileux (Fig. 4), Cette rupture des grains évolue avec l'énergie de compactage (Fig. 5). Il est évident que l'intensité du compactage est un facteur prépondérant sur le développement des ruptures des grains. A chaque augmentation de l'énergie de compactage (nombre de coups appliqué), les courbes granulométriques, après essai, deviennent de plus en plus étalées et s'éloignent de la courbe granulométrique initiale avant essai. Le schiste le plus résistant au compactage est le schiste tacheté, ce qui confirme les constatations lors des essais d'identification qui montrent qu'effectivement il est le plus dur et le moins sujet à la fragmentation des trois schistes étudiés. En effet, les grains sont difficiles à briser et présentent des angularités les plus résistantes. Tandis que le schiste le plus fragile, donc, le moins résistant au compactage est le schiste argileux. En présence d'eau, il y a eu un écrasement plus important des grains sous des énergies de compactage élevées. Ainsi nous pouvons conclure que la présence d'eau fragilise les grains et fait diminuer la résistance à la fragmentation sous les chocs lors du compactage. L'influence de la teneur en eau n'est pas très significative dans le cas d'un matériau résistant schiste satiné (Fig. 6). Cependant l'étalement granulométrique devient de plus en plus important en passant du schiste tacheté au schiste satiné et au schiste argileux (Fig. 4). Donc, son influence évolue en fonction de l'étalement granulométrique des différents matériaux. Le matériau à teneur en eau optimale, dans le cas du schiste argileux, présente des courbes granulométriques proches de celles de l'état sec. Dès que la teneur en eau passe au-delâ de l'optimum Proctor, l'écrasement devient plus important (Fig. 5).

Pour l'essai CBR, les schistes satiné et tacheté, à teneur en eau optimale, présentent des courbes granulométriques proches de celles de l'état sec. Dans le cas du schiste argileux, l'étalement granulométrique à teneur en eau optimale est très important par rapport à celui de l'état sec (Fig. 8). Dès que la teneur en eau passe de part et d'autre de $\mathrm{W}_{\text {urr }}$ on obtient des courbes granulométriques de part et d'autre de celle de l'optimum Proctor. Ceci confirme que l'étalement granulométrique devient de plus en plus important avec l'augmentation de la teneur en eau et donc le taux d'écrasement augmente avec la teneur en eau.

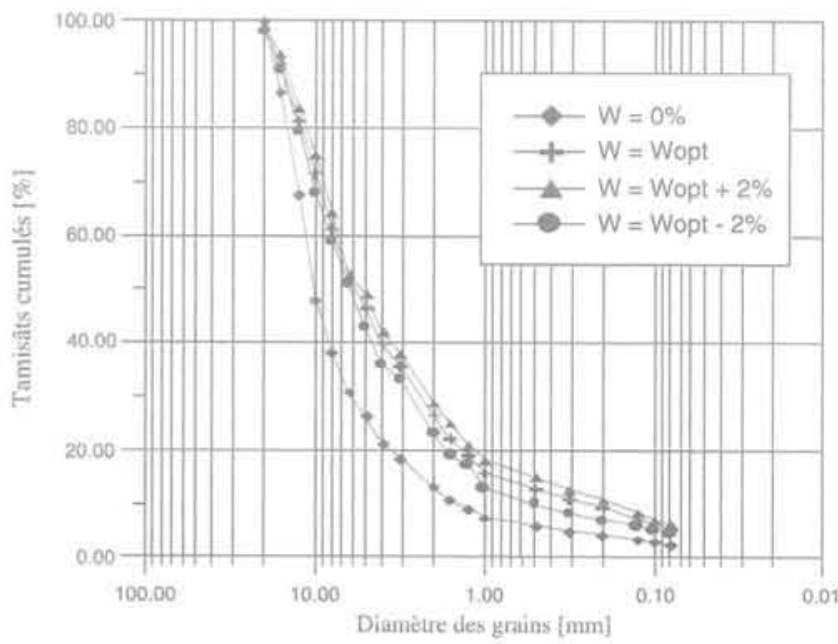

FiG.8 Influence de la teneur en eau sur le degré d'écrasement à l'essai CBR. 


\section{Influence du mode de compactage}

Deux types d'essais ont été retenus pour l'étude de cette influence: l'essai Proctor modifié et l'essai CBR. On a confectionné des échantillons aux deux états : sec lâche et humide à teneur en eau optimale. Les résultats montrent que, pour des énergies assez proches, les matériaux compactés statiquement créent plus de ruptures de grains que ceux compactés dynamiquement, c'est-à-dire le degré d'écrasement à l'essai CBR est plus important que celui de l'essai Proctor que ce soit à l'état sec lâche ou à l'état humide dans le cas des schistes satiné et argileux (Fig. 9). Les courbes granulométriques obtenues sont évidemment plus étalées pour le schiste argileux que pour le schiste satiné. Cet étalement est accentué à l'essai CBR et ceci est justifié par le cumul des deux effets : effet du compactage et du poinçonnement.

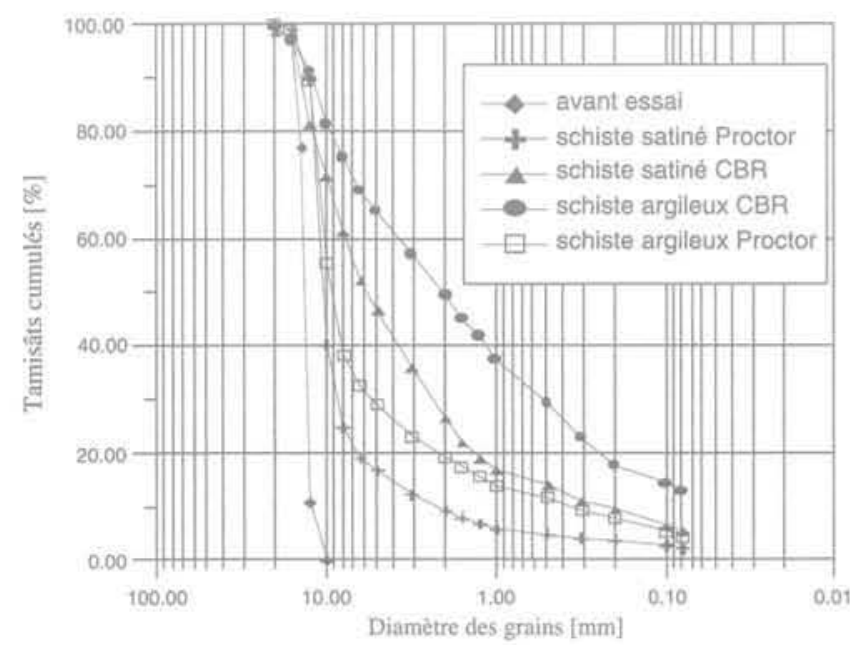

FiG.9 Comportement des schistes argileux et satiné à $W_{o p t}$ à l'essai Proctor et à l'essai CBR.

\section{5}

\section{Quantification de la rupture des grains}

Plusieurs auteurs ont défini le taux d'écrasement des grains à partir de l'êvolution ou de l'étalement de la courbe granulométrique. Certains ont proposé de suivre l'évolution de certains diamètres avant et après essai tels le suggèrent Lade et Yamamuro et al. (1996) pour le diamètre $\mathrm{D}_{1}$. Dans notre cas, pour quantifier la rupture des grains des différents matériaux étudiés aux essais Proctor, deux paramètres ont été retenus: - d'une part. le coefficient d'uniformité $C_{\mathrm{u}}$ :

$$
C_{u}=\frac{D_{60}}{D_{10}}
$$

$\mathrm{D}_{60}$ et $\mathrm{D}_{10}$ sont les diamètres qui correspondent respectivement à $60 \%$ et à $10 \%$ des tamisâts cumulés;

- d'autre part, le facteur $B_{10}$.

Lade et Yamamuro et al. (1996) ont défini le paramètre $B_{10}$ par la relation:

$$
B_{10}=1-\frac{D_{10 f}}{D_{105}}
$$

où $\mathrm{D}_{10}$ et $\mathrm{D}_{10 \mathrm{f}}$ sont les diamètres quil correspondent à $10 \%$ des tamisâts cumulés respectivement avant et après essai. Ainsi $\mathrm{B}_{10}$ est compris entre 0 et 1 . Plus $\mathrm{B}_{10}$ se rapproche de 1, plus il y a des ruptures de grains.

Le paramètre $C_{u}$ montre bien que l'écrasement évolue avec la taille des grains, particulièrement avec les classes granulaires retenues 0/6, 6/10 et 10/14 (Fig. 10), mais aussi avec l'énergie de compactage. $C$ n'évolue que très peu pour le cas de la classe $0 / 6$, ce qui confirme que les grains les plus petits sont les plus résistants. Ce résultat a été aussi confirmé pour les trois matériaux: schistes satiné, argileux et tacheté.

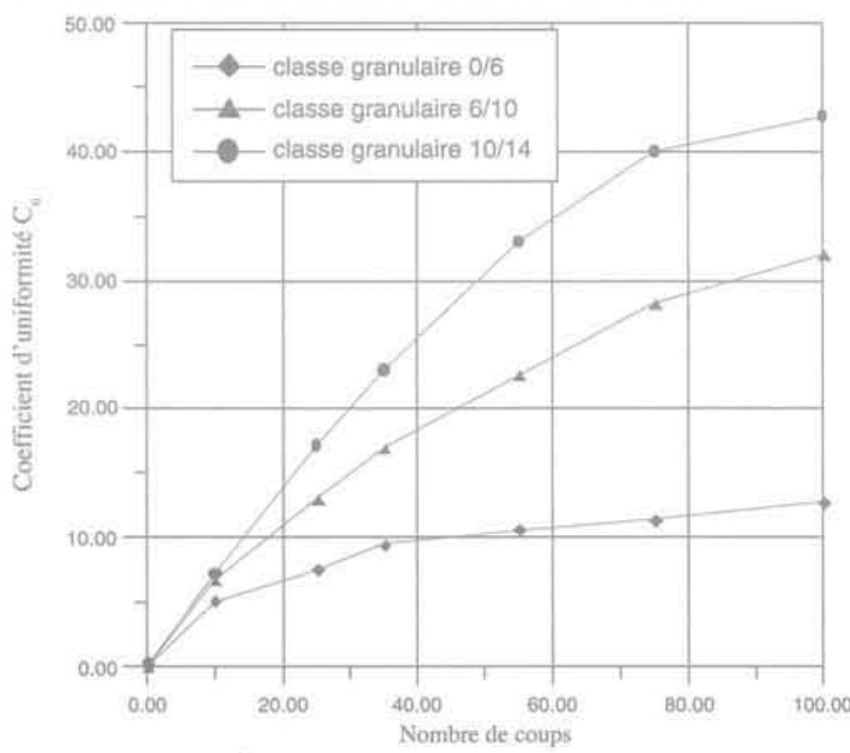

96.10 Évolution du coefficient $\mathrm{C}_{\mathrm{u}}$ en fonction de l'énergie de compactage du schiste argileux à l'état humide pour trois classes granulaires.

A l'état humide $\left(W_{\text {pon }}\right), C_{1}$ montre que la dégradation des grains de schiste satiné de la classe 10/14 est très importante comparativement aux classes $6 / 10$ et $0 / 6$. Elle évolue en fonction de l'énergie de compactage (Fig. 11). Les gros grains présentent des angularités et certainement des fissures où la pénétration d'eau peut,

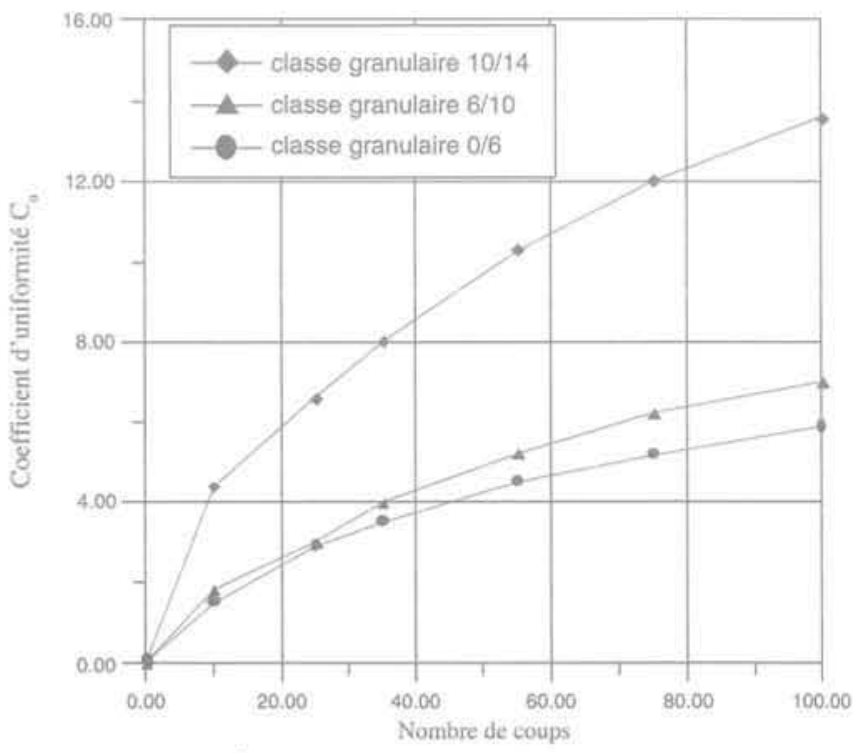

FiG. 11 Évolution du coefficient $C$ en fonction de l'énergie de compactage du schiste satiné à l'état humide pour les trois classes granulaires. 
s'effectuer et éventuellement favoriser leur rupture. La figure 12 montre que $C_{\text {e }}$ évolue de façon identique en fonction du nombre dé coups dans le cas des deux schistes tacheté et satiné à l'état sec.

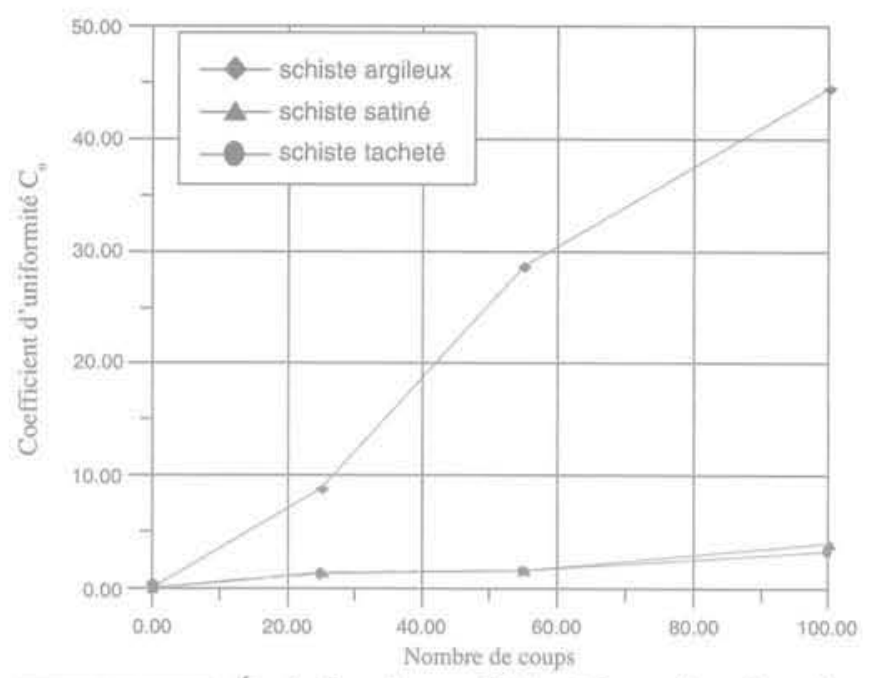

FIG. 12 Évolution du coefficient $\mathrm{C}_{4}$ en fonction de l'énergie de compactage pour les trois matériaux à l'état sec.

Pour la classe $0 / 6$, à l'état sec, le facteur $\mathrm{B}_{10}$ évolue en fonction du nombre de coups de 0 à 0,36 pour le schiste satiné, de 0 à 0,32 pour le schiste tacheté et de 0 à 0,69 pour le schiste argileux (Fig. 13). On note que l'évolution des schistes satiné et tacheté est quasi identique et proche l'une de l'autre. Par contre à l'état humide, $\mathrm{B}_{10}$ évolue de 0 à 0,70 pour le schiste tacheté, de 0 à 0,73 pour le schiste satiné et de 0 à 0,77 pour le schiste argileux (Fig. 14). Cette évolution du facteur $B_{10}$ s'accentue avec la taille des grains (Fig. 15) et avec les minéraux constituants chaque matériau. En effet $B_{10}$ est de l'ordre de 0,98 pour le schiste argileux qui présente les minéraux les plus instables; alors qu'il est égal à 0,86 pour le schiste satiné et à 0,82 pour le schiste tacheté.

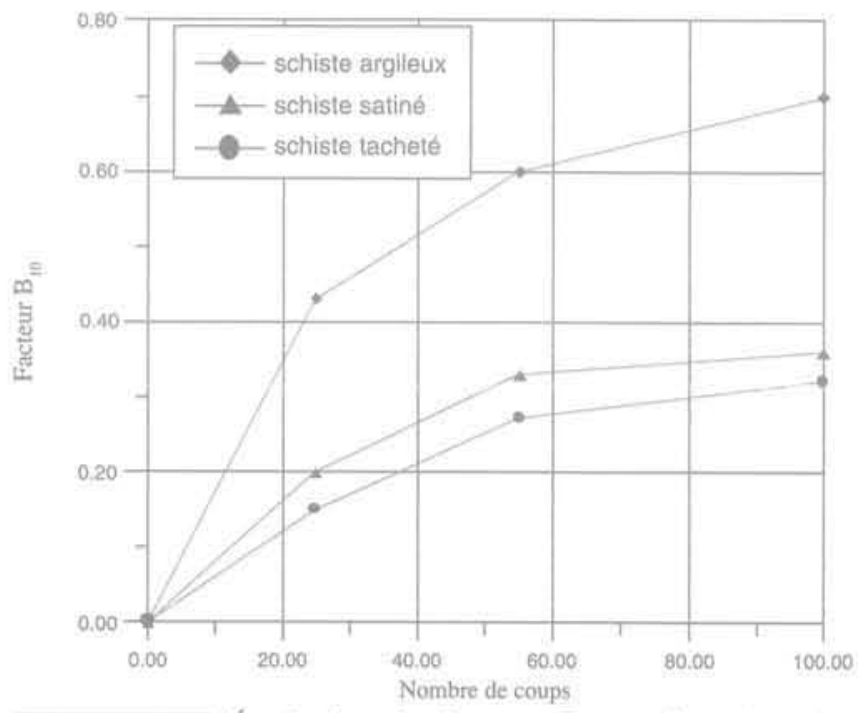

FIG. 13 Evolution du facteur $B_{10}$ en fonction de l'énergie de compactage pour les trois matériaux de classe $0 / 6$ à l'état sec.

Le paramètre $\mathrm{C}_{\mathrm{u}}$ confirme que le degré d'écrasement des grains est moindre pour le schiste tacheté qui est le matériau le plus résistant que pour les deux autres matériaux schisteux satiné et argileux. Le facteur

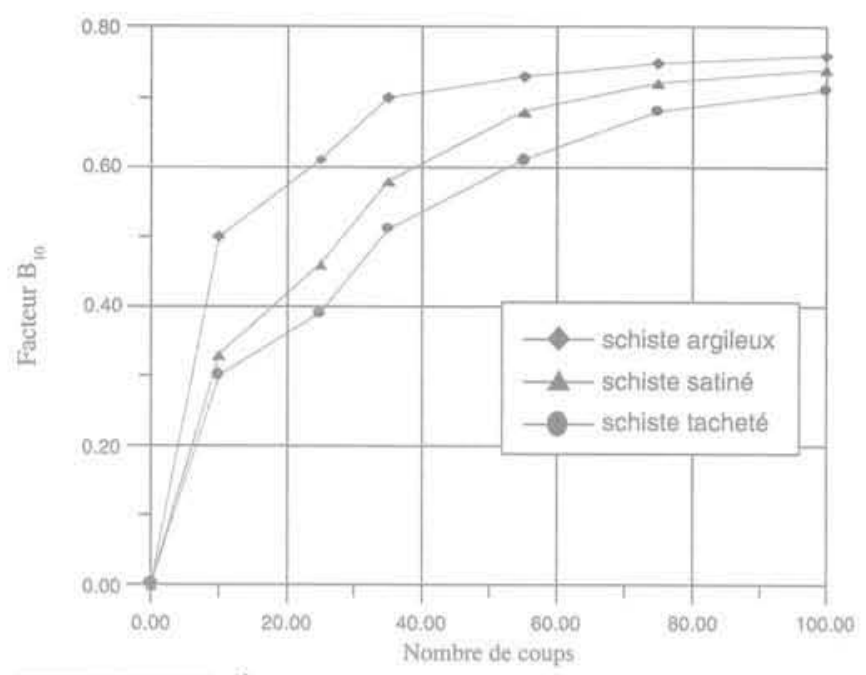

FIG.14 Évolution du facteur $B_{10}$ en fonction de l'énergie de compactage pour les trois matériaux de classe $0 / 6$ à l'état humide.

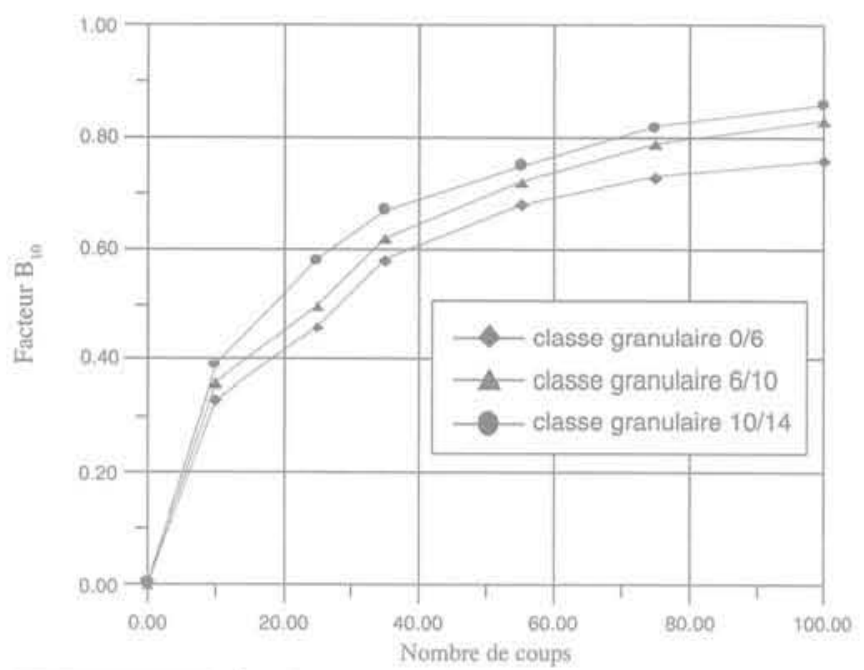

FIG. 15 Évolution du facteur $B_{10}$ en fonction de T'énergie de compactage pour les trois classes granulaires du schiste satiné à Y'état humide.

$B_{10}$ montre que l'écart du degré de rupture, qui évolue en fonction de l'énergie de compactage, entre les classes granulaires n'est pas très important (Fig. 15).

Pour la classe 10/14, le coefficient d'uniformité $C_{4}$ et le facteur $B_{10}$ évoluent en fonction de la teneur en eau. $C_{1}$ varie de 40,00 à 62,86 pour le schiste argileux, de 5,45 à 7,69 pour le schiste tacheté et de 6,25 à 9,81 pour le schiste satiné (Fig. 17), Le facteur $\mathrm{B}_{10}$ a atteint 0,98 pour le schiste argileux alors qu'il vaut 0,87 pour le schiste satiné et 0,85 pour le schiste tacheté (Fig. 17). Le coefficient $C_{4}$ est lié à la teneur en eau de façon quasi proportionnelle particulièrement pour les schistes satiné et tacheté mais avec une faible pente; alors que le facteur $B_{40}$ est lié à la teneur en eau $W$ avec une pente légèrement élevée pour ces deux derniers matériaux. Pour le schiste argileux, la pente est très faible et $\mathrm{B}_{10}$ à l'état sec est de l'ordre de 0,97 , ce qui montre qu'à cet état la presque totalité des grains s'est écrasée. 


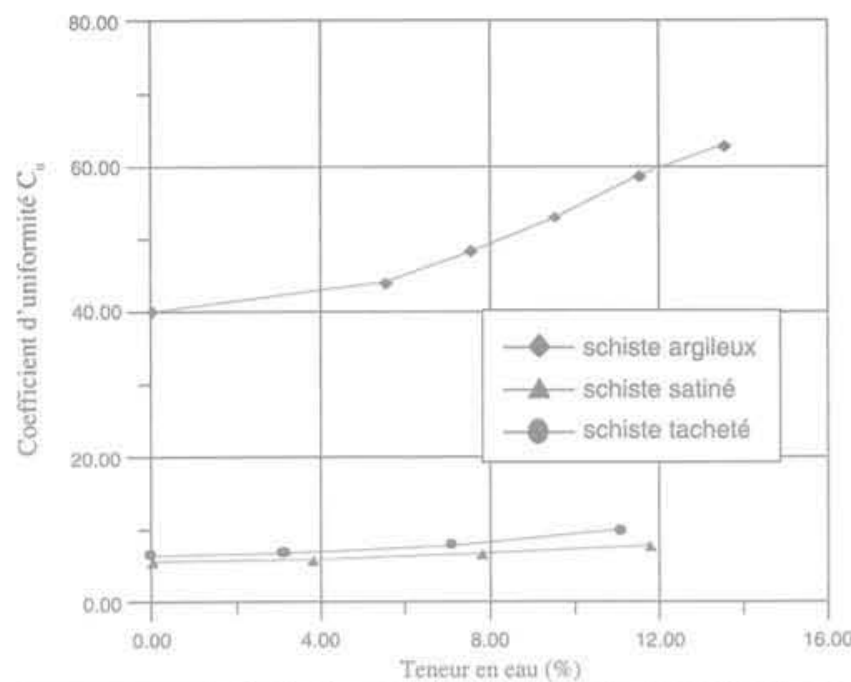

FIG.16 Évolution du coefficient d'uniformité $\mathrm{C}_{u}$ en fonction de la teneur en eau pour une énergie de compactage 100 coups.

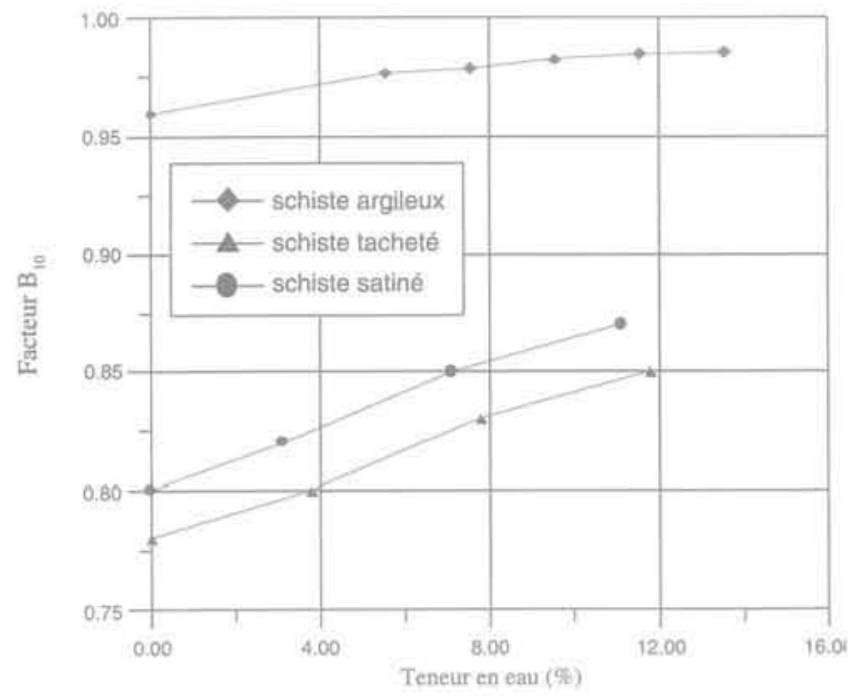

FG. 17 Évolution du facteur $B_{40}$ en fonction de la teneur en eau pour une énergie de compactage de 100 coups.

\section{Conclusion}

Ces essais ont montré que l'écrasement des grains augmente considérablement avec la fragilité de ces derniers constituant chacun des schistes. Ce phénomène semble être lié en grande partie à la minéralogie, à la texture, à l'angularité des grains et au pourcentage des minéraux instables.

La résistance au compactage des schistes est liée aussi à la nature minéralogique des grains, à la granulométrie (taille des grains), à l'intensité des efforts appliqués (nombre de coups) et à la compacité de l'échantillon: lâche, dense (densité à l'optimum Proctor) avec ou sans présence d'eau.

Les schistes argileux, les plus fragmentables des trois types de schistes étudiés, ont généré les courbes granulométriques les plus étalées après essai, celles-ci correspondent non seulement à des écrasements mais conduisent aussi à des glissements et à des arrangements des grains vers des indices des vides petits, par conséquent, des degrés d'écrasement les plus importants.

La dureté des minéraux, telle celle de l'amphibole, diminue la quantité d'écrasement des grains. Les échantillons constitués de minéraux les plus durs, même soumis à des efforts élevés, donnent peu de rupture.

Le coefficient d'uniformité $\mathrm{C}_{4}$ et le facteur $\mathrm{B}_{10}$ confirment que la dégradation des grains est plus importante pour les grains de grande taille, leurs valeurs sont plus élevées à l'état humide qu'à l'état sec et sont fonction de la minéralogie des matériaux.

\section{Bibliographie}

Biarez J., Hicher P.Y. - Influence de la granulométrie et de son évolution par ruptures de grains sur le comportement mécanique de matériaux granulaires. Revue française de génie civil, vol. 1 . $n^{\circ} 4 / 1997,1997$, p. 607-631.

Cambou B. - Compressibilité d'un milieu pulvérulent, influence de la forme et de la dimension des particules sur les propriêtés mécaniques d'un millieu pulvérulent. Thèse de docteur de spécialité, université scientifique et médicale, Grenoble, 1972.

Guyon E., Troadec J.-P. - Du sac de billes au tas de sable. Editions Odile Jacob. Sciences, 1994.
Hagerty M.M., Hite D.R., Ulirich C.R., Hagerty D.J. - One dimensional high pressure compression of granular media. Journal of Geotechnical Engineering, vol. 119, $\mathrm{n}^{\circ}$ 1, January 1993 , p. $1-17$.

Lade P.V., Yamamuro J.A., et al, - Significance of particle crushing in granular materials, Journal of Geotechnical Engineering, vol. 122, n 4. 1996, p. 31093116.

Marsal R.J. - Research on granular materials. Experimental work compiled for the IXth International Conference on Soils. Mechanics and Foundation, Engineering. June, Tokyo, 1977, p. 1-78.
McDowell G.R., Bolton M.D. - On the micromechanics of crushable aggregates. Geotechnique $48, n^{\circ} 5,1998$, p. $667-679$

Miura N., O' Hara S. - Particle-crushing of a decomposed granite soil under shear stresses. Soils and Foundations, vol. 19, $n^{\circ} 3,1979$, p. 1-14.

Ramamurthy T. - Crushing phenomena in granular soils. Journal of the Indian National Society of Soil Mechanics and Foundation Engineering, vol. 8, 1969, p. $67-86$.

Yamamuro J.A., Bopp P.A., Lade P.V. - One dimentional compression of sands at high pressures. Journal of Geotechnical Engineering, February, 1996, p. 147-154. 